\title{
Design of a Road Friendly SAS System for Heavy-Duty Vehicles Based on a Fuzzy-Hybrid-ADD and GH-Control Strategy
}

\author{
Jing Zhao, Pak Kin Wong, Zhengchao Xie, Xinbo Ma, and Caiyang Wei \\ Department of Electromechanical Engineering, University of Macau, Macau \\ Correspondence should be addressed to Zhengchao Xie; zxie@umac.mo
}

Received 9 July 2015; Accepted 9 September 2015

Academic Editor: Wen-Hsiang Hsieh

Copyright ( 2016 Jing Zhao et al. This is an open access article distributed under the Creative Commons Attribution License, which permits unrestricted use, distribution, and reproduction in any medium, provided the original work is properly cited.

\begin{abstract}
Semiactive suspension (SAS) system has been widely used for its outstanding performance in offering competent ride quality, road holding, and handling capacity. However, the road friendliness is also one of the crucial factors that should be attached in the design of the SAS system for heavy-duty vehicles. In this study, a fuzzy controlled hybrid-acceleration driven damper (ADD) and ground hook- (GH-) control strategy is proposed for SAS system of heavy-duty vehicles. Firstly, a quarter-vehicle model with SAS system is constructed. Then, aiming to improve the ride quality and road friendliness, a hybrid-ADD and GH-control strategy is proposed under the coordination of the fuzzy controller. Numerical results show that the ride quality and road friendliness of the SAS system with the proposed control strategy outperform those with traditional hybrid-sky hook and ground hook-control strategy. It is also verified that the proposed strategy is superior to the sole ADD approach and sole ground hook approach in improving the vehicle overall performance.
\end{abstract}

\section{Introduction}

In the past decades, ever increasing requirement in commercial transportation inevitably accelerates the maintenance cycle of the road (e.g., frequently overloading of the building materials in commercial vehicles accelerates the rate of pavement damage) [1]. However, regarding the probability of road damage coming from commercial vehicles, the heavyduty vehicle accounts for a large percentage and is responsible for most of the road damage due to its huge axle load. The heavy-duty vehicle has been prevalently used and it cannot be replaced in commercial transportations due to its high loading capacity and low freight rate. So, taking into account the cost of road maintenance, this work aims to design a road friendly suspension system for heavy-duty vehicles so that the road damage coming from heavy-duty vehicles can be reduced.

Road friendliness refers to the extent of damage exerted on the road by the vehicles, and it can be quantified by the dynamic tire load (DTL) of the vehicle. The design of the road friendly suspension system can be regarded as an effective method to improve the road friendliness of the vehicle.
A well-designed road friendly suspension should be capable of decreasing the DTL and minimizing the road damage coming from the vehicle. With respect to the vehicle DTL, it is commonly accepted that by adopting proper suspension system the vehicle DTL can be effectively declined.

As far as the suspension systems are concerned, the semiactive suspension (SAS) system has been considered as the most popular type for heavy-duty vehicles as it can provide an achievable performance between the active suspension system and passive suspension. Moreover, the low power consumption and fail-safe property of the SAS system also determine that it is applicable for heavy-duty vehicles. As a result, a SAS system is utilized in the design of the road friendly suspension system for heavy-duty vehicles.

In addition to the road friendliness, another concern worth mentioning for heavy-duty vehicles is the vertical acceleration. During the transportation, the goods in the rear part of heavy-duty vehicles probably suffer a fluctuation along with the sprung mass acceleration (SMA) of the vehicle, and this may lead to certain damage to the goods. To protect the goods from damaging, the SMA of heavy-duty vehicles should be minimized and stabilized within a certain range. 
With the above considerations, the objective of this paper is to propose a SAS system for heavy-duty vehicles to reduce the DTL and SMA simultaneously. To accomplish this target, of great concern is the control strategy of the SAS system, because the performance of the SAS system is largely influenced by the control strategy applied in its dissipation device. For most of the heavy-duty vehicles, the dissipation device of the SAS system is an adjustable damper. Therefore, a suitable control strategy must be selected for the adjustable damper of the SAS system.

Various investigations have been done for the control strategies of the adjustable damper. To control the SMA, the most widely employed strategy is the sky hook ( $\mathrm{SH}$ ) approach, which is first proposed by Karnopp et al. [2]. The idea of the $\mathrm{SH}$ approach is to attach the vehicle chassis to the sky via an active damping force. By this way, the vibration from the vehicle chassis can be reduced accordingly. It has been verified by many studies as a simple but effective way to reduce the SMA [3-5]. In addition, another effective approach for SMA control is the acceleration-driven damper (ADD) control strategy [6, 7]. This approach has been proved to be the optimal one in the minimization of the SMA and also more simple in the aspect of implementation than the $\mathrm{SH}$ approach.

When it turns to the control of the vehicle DTL, some of the existing investigations are dedicated to the study of the opposite side of the SH approach, namely, ground hook $(\mathrm{GH})$ approach $[8,9]$. The GH approach focuses on increasing the suspension damping and reducing the DTL together. Furthermore, it is also reported that the $\mathrm{H}$ infinity control is also favorable for the control of the DTL [10]. However, when applying the $\mathrm{H}$ infinity control to the adjustable damper of the SAS system, a constraint should be set for the adjustable damper in advance and as well a saturation should also be considered for the damping force. The inaccuracy that comes from the constraint and complicated saturation setting make the $\mathrm{H}$ infinity approach popular in some active suspension system but the SAS system.

It should be noted that the single application of any of the above approaches cannot enable the well design of the SAS system (i.e., impossible to improve the SMA and the DTL simultaneously). So, the hybrid strategy, in which the individual control approaches are combined to achieve different potential targets, is proposed, such as hybrid optimal control and hybrid integrated control [11, 12]. Within the hybrid control strategy, a tuning method should be well defined for different individual control approaches and then a promising performance can be obtained. As far as the tuning method is concerned, the fuzzy logic control has been proved to be effective in the tradeoff of two different control objectives [13-15], and it is also employed in this work.

Up to this point, a fuzzy controlled hybrid-ADD and GHcontrol strategy is proposed for the SAS system. Moreover, a traditional hybrid-SH and $\mathrm{GH}$-control which uses a straightforward tuning method is also introduced as a comparative case so as to highlight the superiority of the proposed hybrid fuzzy tuning method. Furthermore, the sole ADD approach and the sole $\mathrm{GH}$ approach are also selected as the comparative cases. The rest of this paper is formatted as follows. Section 2

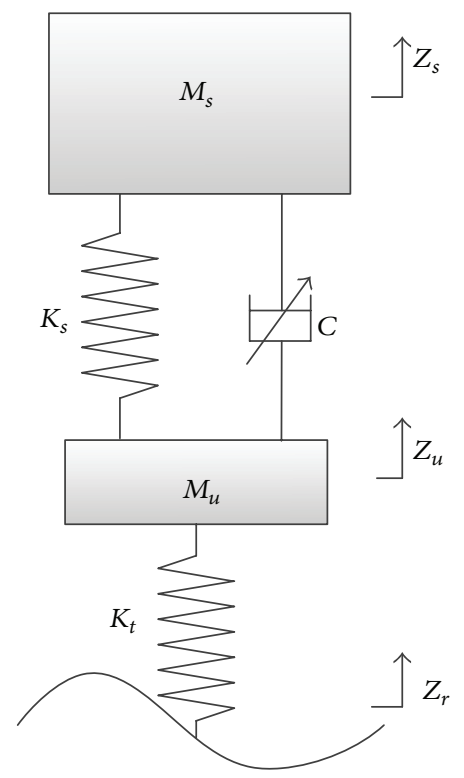

FIGURE 1: Quarter-vehicle model with semiactive suspension system.

introduces the modeling of the quarter vehicle. The presentation of the proposed control strategy and the comparative cases are illustrated in Section 3. Section 4 gives numerical results and analysis, and conclusion is provided in Section 5.

\section{Modeling}

To examine the proposed control strategy, a vehicle model should be constructed first. Generally speaking, the rear suspension undertakes the main load of heavy-duty vehicles, so the goods damage as well as the road damage is often seriously found in the rear part of heavy-duty vehicles. In view of this point, the model of the rear suspension system is chosen to study proposed control strategy.

The objective of this work is to control the SMA and the DTL of heavy-duty vehicles, and 2 degrees of freedom (DOF) vehicle model is capable of examining the above control objectives. Therefore, a quarter-vehicle model is employed in this work. The schematic diagram of the quarter-vehicle model with SAS system is shown in Figure 1.

Moreover, it is also important to describe the equation of motions for the quarter-vehicle model so that the control input and output can be presented more clearly. For the quarter-vehicle model, the vehicle motions in vertical direction can be written as

$$
\begin{aligned}
& M_{s} \ddot{Z}_{s}=C\left(\dot{Z}_{u}-\dot{Z}_{s}\right)+K_{s}\left(Z_{u}-Z_{s}\right), \\
& M_{u} \ddot{Z}_{u}=C\left(\dot{Z}_{s}-\dot{Z}_{u}\right)+K_{s}\left(Z_{s}-Z_{u}\right)-K_{t}\left(Z_{u}-Z_{r}\right),
\end{aligned}
$$

where $m_{s}$ and $m_{u}$ are the sprung mass and unsprung mass, respectively, $k_{t}$ is the stiffness of the tire, $k_{s}$ is the stiffness of the spring, $C$ is the damping coefficient of the damper, $Z_{r}$ is the road input, and $Z_{u}$ and $Z_{s}$ are the displacement of 
TABle 1: Parameters for modeling.

\begin{tabular}{lcc}
\hline Item & Value & Unit \\
\hline$M_{s}$ & 3257 & $\mathrm{Kg}$ \\
$M_{u}$ & 312 & $\mathrm{Kg}$ \\
$K_{s}$ & $3.5 \times 10^{5}$ & $\mathrm{KN} / \mathrm{m}$ \\
$K_{t}$ & $3 \times 10^{6}$ & $\mathrm{KN} / \mathrm{m}$ \\
$G\left(n_{0}\right)$ & $2.56 \times 10^{-6}$ & $/$ \\
$f_{0}$ & 72 & $\mathrm{~Hz}$ \\
$v$ & 20 & $\mathrm{~m} / \mathrm{s}$ \\
$w(t)$ & 1 & $/$ \\
\hline
\end{tabular}

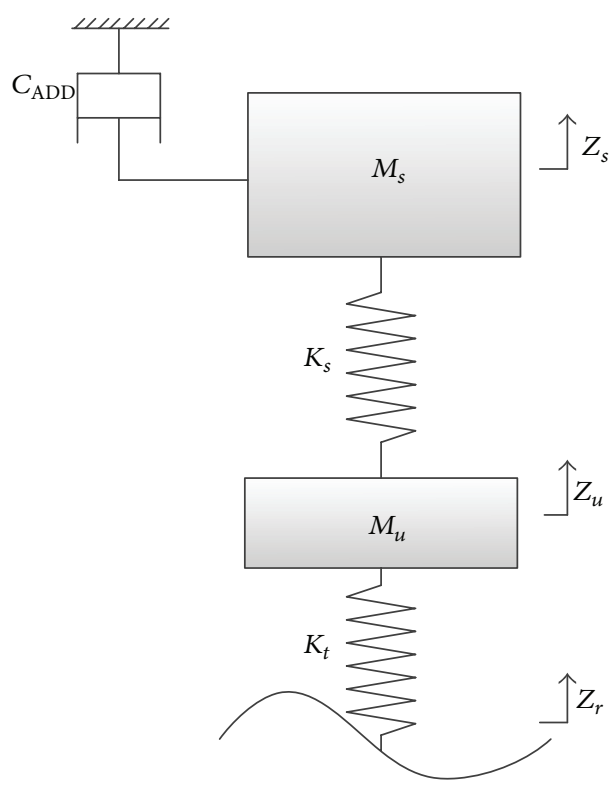

FIGURE 2: Quarter-vehicle SAS system model with ADD approach.

the unsprung mass and displacement of the sprung mass, respectively.

By this means, the quarter-vehicle model with SAS system can be constructed with the parameters in Table 1. Also, the SAS system with ADD approach and GH approach is also created in the quarter-vehicle model, as shown in Figures 2 and 3 , respectively.

To further conduct the numerical analysis, a road input should be provided as the excitation of the tire. In this connection, a band-limit white noise method is utilized as the power source of the road profile and a C-class level road pavement is chosen as the road excitation of the tire. The road input of the vehicle tire can be presented as

$$
\dot{Z}_{r}(t)=-2 \pi f_{0} Z_{r}(t)+2 \pi \sqrt{G_{0}(n) v} w(t),
$$

where $f_{0}$ is the lower cut-off frequency, $G_{0}(n)$ is the road roughness coefficient, $v$ is the vehicle speed, and $w(t)$ is the signal power of the white noise. Then, with the parameters in Table 1, the road excitation can be obtained, as shown in Figure 4.

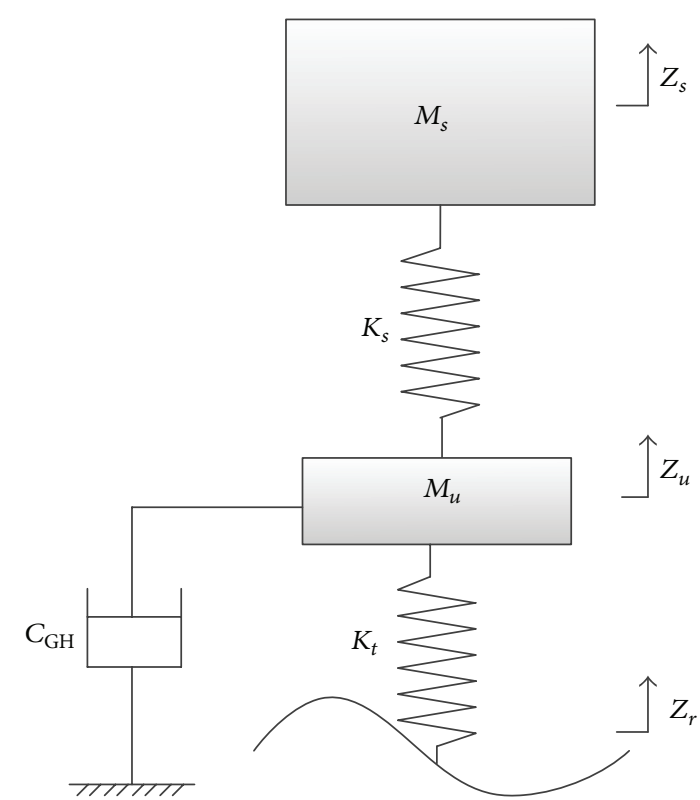

FIGURE 3: Quarter-vehicle SAS system with GH approach.

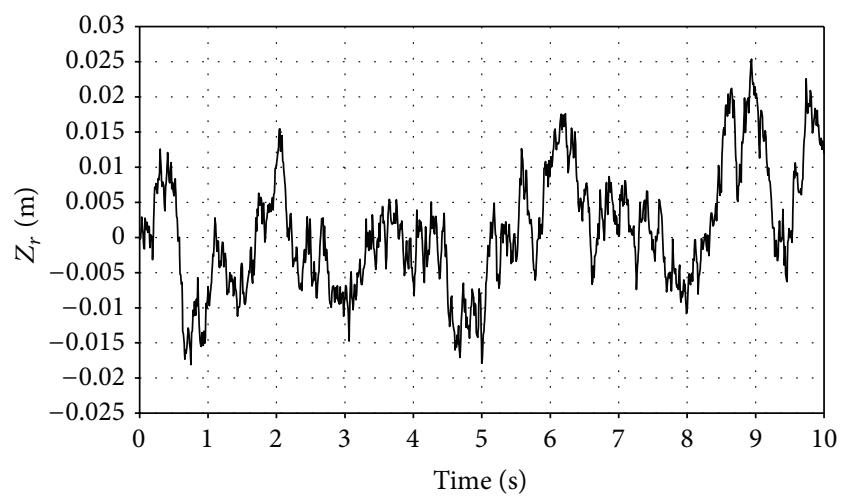

FIgURE 4: Road excitation.

\section{Control Strategies}

This section describes the design process of the proposed control strategy. Also, to highlight the performance of the proposed control strategy, some comparative cases such as sole ADD approach, sole GH approach, and a straightforward tuning hybrid-SH and GH-control strategy are also introduced. Regarding the control of the SAS system, of particular concern is the control of the damping force. So, for each of the above cases, the control process regarding the damping force is discussed, as shown below.

Case 1 (ADD approach). The ADD approach is improved on the basis of the $\mathrm{SH}$ approach and it mainly focuses on the control of the SMA. The ADD approach takes the state of the sprung mass as its control variables and then switches the damping force with the various states of the sprung mass. The configuration of the ADD approach for the SAS system is shown in Figure 2. 


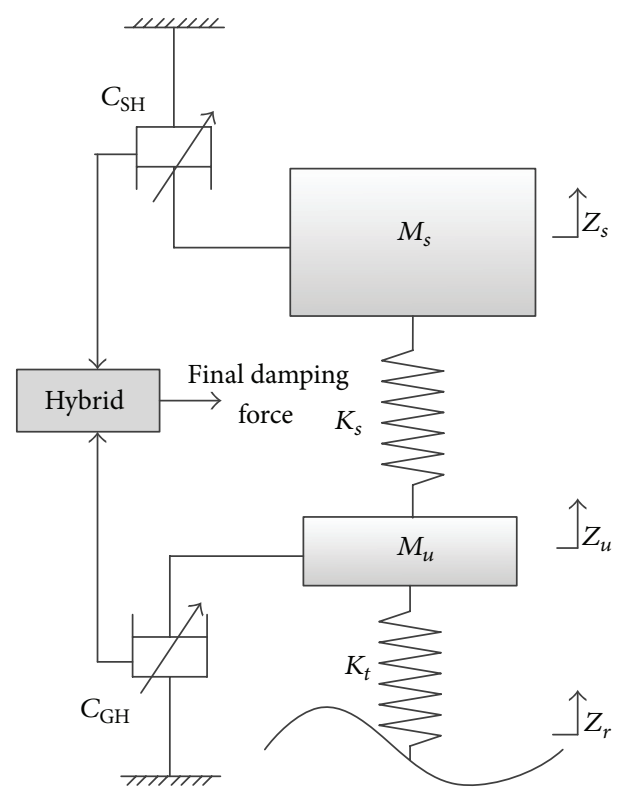

FIGURE 5: Quarter-vehicle SAS system model with hybrid-SH and GH-control.

The damping force of the SAS system with ADD approach can be represented as

$$
F_{\mathrm{ADD}}= \begin{cases}C_{\mathrm{ADD}} \dot{Z}_{s}, & \text { when } \ddot{Z}_{s}\left(\dot{Z}_{s}-\dot{Z}_{u}\right) \geq 0 \\ 0, & \text { when } \ddot{Z}_{s}\left(\dot{Z}_{s}-\dot{Z}_{u}\right)<0,\end{cases}
$$

where $C_{\mathrm{ADD}}$ is the damping coefficient of the SAS system, $\dot{Z}_{s}$ is the absolute velocity of sprung mass, $\ddot{Z}_{s}$ is the absolute acceleration of sprung mass, and $\dot{Z}_{u}$ is the absolute velocity of unsprung mass.

Case 2 (GH approach). The GH approach can be viewed as an opposite side of the ADD approach and it mainly concerns the control of the tire deformation. By reducing the tire deformation, the dynamic tire load can be decreased. The $\mathrm{GH}$ approach considers the state of the unsprung mass in its control process, and the schematic diagram of the GH approach in the SAS system is illustrated in Figure 3.

The damping force of the SAS system with GH approach can be described as

$$
F_{\mathrm{GH}}= \begin{cases}C_{\mathrm{GH}} \dot{Z}_{u}, & \text { when } \dot{Z}_{u}\left(\dot{Z}_{s}-\dot{Z}_{u}\right) \leq 0 \\ 0, & \text { when } \dot{Z}_{u}\left(\dot{Z}_{s}-\dot{Z}_{u}\right)>0,\end{cases}
$$

where $C_{\mathrm{GH}}$ is the damping coefficient of the SAS system.

Case 3 (traditional hybrid-SH and GH-strategy). The traditional hybrid-SH and GH-strategy introduced in this work is a straightforward hybrid tuning method between the $\mathrm{SH}$ and the GH approach, as shown in Figure 5. The damping force of the SAS system with this method can be represented as

$$
F_{\mathrm{HSG}}=\beta F_{\mathrm{SH}}+(1-\beta) F_{\mathrm{GH}} \text {, }
$$

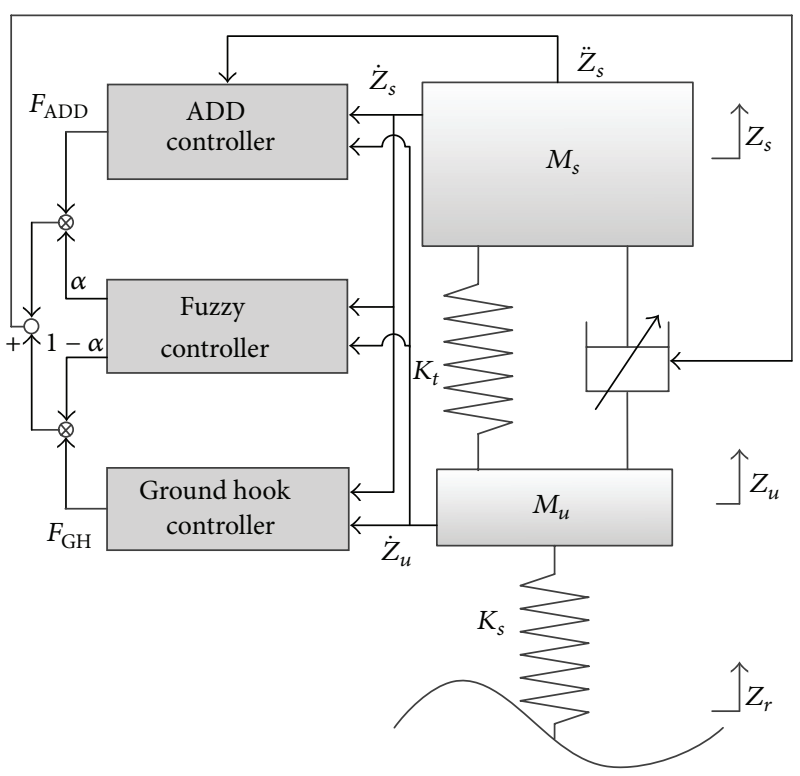

FIGURE 6: Quarter-vehicle SAS system model with fuzzy-hybrid$\mathrm{ADD}$ and GH-control.

where $\beta$ is the weighting coefficient used for determining the contribution of the SH approach and the GH approach to the final damping force. With different settings of the $\beta$ values, various suspension performances can be achieved. To simplify the calculation, $\beta$ is defined as $\beta \in[0,1]$. Furthermore, in light of the balance between the SMA and DTL, $\beta$ is set as 0.5. $F_{\mathrm{GH}}$ is the damping force with the $\mathrm{GH}$ approach (regarding its control rules, refer to Case 2). Note that $F_{\mathrm{SH}}$ here is the damping force with the sky hook approach and it complies with the following rules:

$$
F_{\mathrm{SH}}= \begin{cases}C_{\mathrm{SH}} \dot{Z}_{s}, & \text { when } \dot{Z}_{s}\left(\dot{Z}_{s}-\dot{Z}_{u}\right) \geq 0 \\ 0, & \text { when } \dot{Z}_{s}\left(\dot{Z}_{s}-\dot{Z}_{u}\right)<0,\end{cases}
$$

where $C_{\mathrm{SH}}$ is the damping coefficient of the SAS system.

Case 4 (proposed fuzzy-hybrid-ADD and GH-strategy). Obviously, the simple tuning method (i.e., the straightforward tuning method for hybrid strategy used in Case 3) between the two approaches cannot meet the variable state of the vehicle dynamics. So, a more suitable tuning method, which can not only combine the two individual control methods but also coordinate between the methods, is more desirable.

The fuzzy logic method is used to communicate and coordinate between the ADD and GH approaches for the proposed control strategy. The schematic diagram of the proposed system with fuzzy controlled hybrid-ADD and GHstrategy is shown in Figure 6. As shown in Figure 6, with the dynamic response of the SAS system, the absolute velocity of the sprung mass and absolute velocity of the unsprung mass are first delivered to the sole ADD controller, the sole GH controller, and the fuzzy controller, respectively. With the received signals, the ADD controller and GH controller 


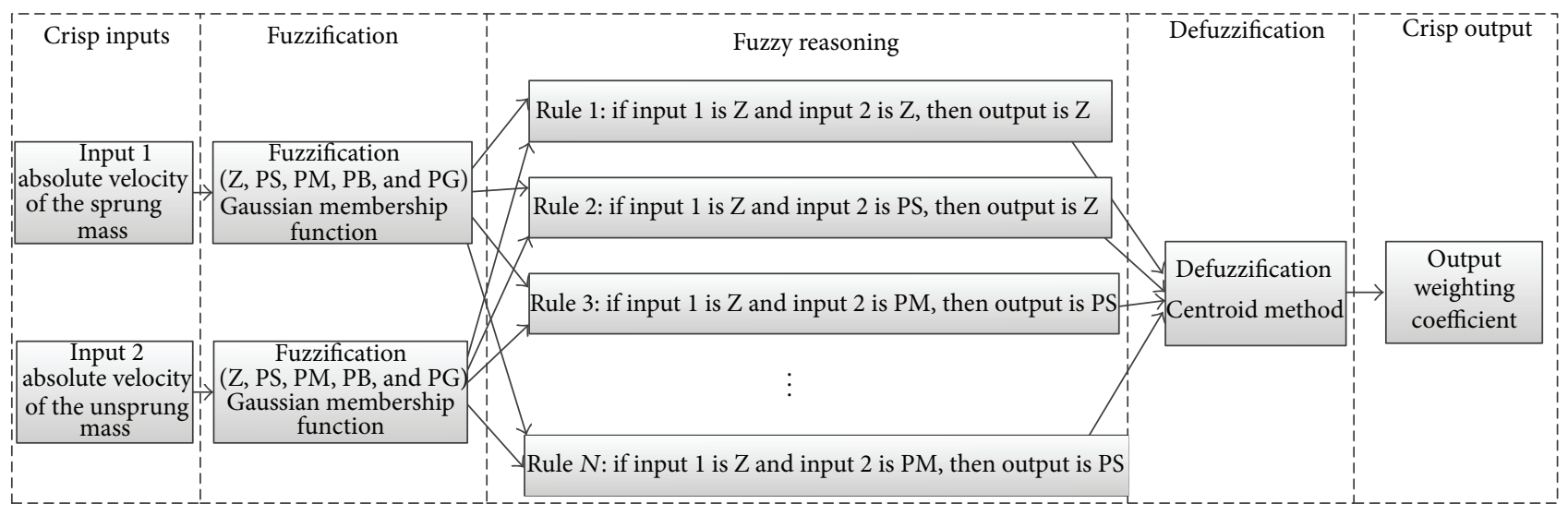

FIgURE 7: Flowchart of the fuzzy controller.

TABLE 2: Rules of the fuzzy controller.

\begin{tabular}{ccccccc}
\hline \multicolumn{3}{c}{$\beta$} & & & & $\dot{Z}_{s}$ \\
& & $\mathrm{Z}$ & PS & PM & PS & PG \\
\hline \multirow{4}{*}{$\dot{Z}_{u}$} & Z & Z & Z & PS & PS & PM \\
& PS & Z & PS & PM & PM & PB \\
& PM & PS & PM & PM & PB & PB \\
& PB & PS & PM & PB & PB & PG \\
& PG & PM & PB & PB & PG & PG \\
\hline
\end{tabular}

compute the corresponding damping force with their own rules. As for the fuzzy controller, the received signal is used to calculate the weighting coefficient relative to the tuning of the hybrid-ADD and GH-strategy. After that, the calculated weighting coefficients are then separately distributed to the $\mathrm{ADD}$ controller and the $\mathrm{GH}$ controller for further processing. By multiplying respective weighting coefficient, the damping force from each of the two approaches (i.e., ADD approach and GH approach) can be computed, and then the final damping force of the proposed system can be obtained.

In the fuzzy controller, there are five parts, including crisp inputs, fuzzification, fuzzy reasoning, defuzzification, and crisp output, as shown in Figure 7. First of all, the absolute velocity of the sprung mass and the absolute velocity of the unsprung mass are regarded as the inputs with crisp numbers. The domains of discourse of them are $[-0.04,0.04]$ and $[-0.025,0.025]$, respectively. Next, the Gaussian function is adopted as the membership function to fuzzify the measured crisp inputs into soft fuzzy variables, and five linguistic variables, Zero "Z," Positive Small "PS," Positive Medium "PM," Positive Big "PB," and Positive Great "PG," are defined. Then, Mamdani logic is utilized as the method of fuzzy reasoning according to the rule base described in Figure 8 and Table 2. After that, defuzzification is carried out by using centroid method to convert the fuzzy value represented by membership function into crisp output. Finally, as the crisp output, the weighting coefficient with the domain of discourse $[0,1]$ is exported.

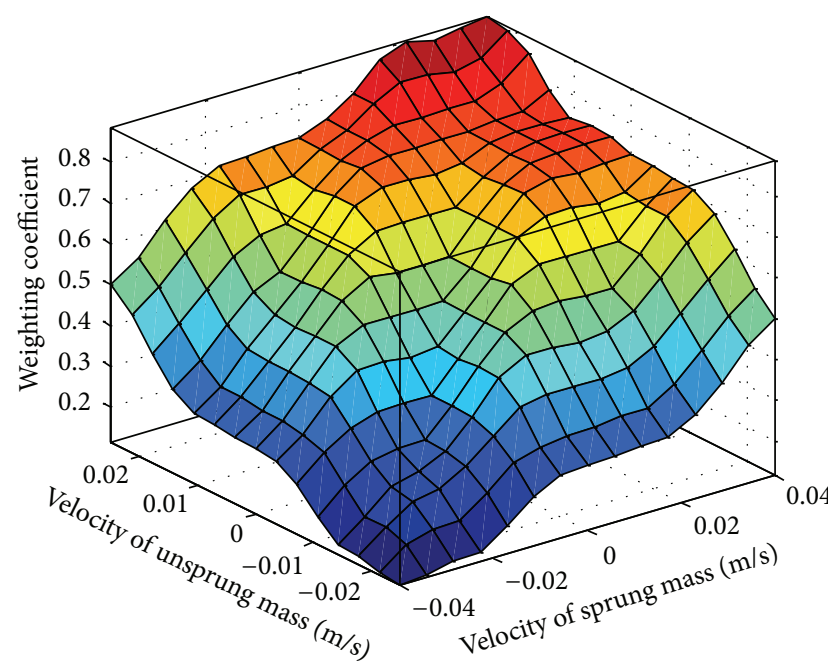

Figure 8: Compositional rule of inference for the fuzzy controller.

\section{Numerical Results and Analysis}

To examine the performance of the proposed strategy, numerical simulations are conducted based on the models constructed in Section 2. The numerical results with various control strategies are shown in Figures 9 and 10. In addition, to clearly figure out the improvement of proposed strategy, the RMS values of the SMA and DTL are also computed for each of the control strategies, as described in Table 3.

Figure 9 shows the SMA with different control strategies. It can be seen that ADD approach achieves the best performance as compared with other cases while GH approach performs worst. However, in Figure 10, the GH approach excels the other strategies while ADD performs worst in the DTL. The reasons for the above results lie in the fact that the ADD approach and GH approach are particularly designed for improving the SMA and DTL, respectively. Moreover, in Figures 9 and 10, the proposed strategy outperforms the traditional hybrid-SH and GH-strategy in terms of the SMA and DTL, which means the fuzzy controller takes effect in the coordination of the two individual approaches. 
TABLE 3: Control performance with various control strategies.

\begin{tabular}{lcccr}
\hline Item & & \multicolumn{2}{c}{ Methods } \\
& ADD & GH & Hybrid-SH and GH & Fuzzy tuning hybrid-ADD and GH \\
\hline RMS of the SMA & $\mathbf{1 . 0 9 9}$ & 2.033 & 1.710 & 1.226 \\
RMS of the DTL & 8347 & $\mathbf{4 3 0 0}$ & 6979 & 5495 \\
Overall performance & 0.471 & 0.423 & 0.590 & $\mathbf{0 . 1 9 6}$ \\
Improvement & $58.39 \%$ & $53.66 \%$ & $66.78 \%$ & $/$ \\
\hline
\end{tabular}

Note: $*$ means the overall performance improvement of the proposed strategy compared to the method of current item.

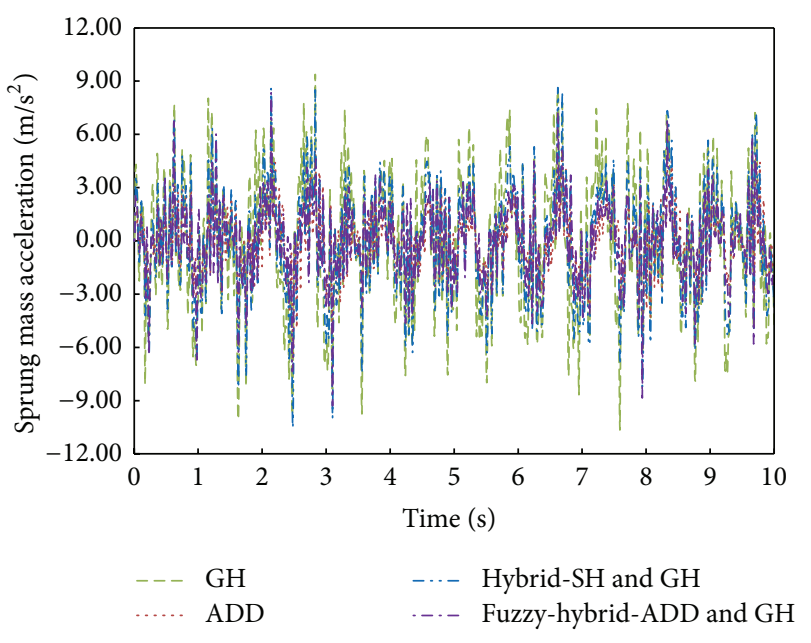

FIGURE 9: Comparison of sprung mass acceleration.

In addition to the comparison with numerical results, a fitness function is designed to further evaluate the improvement of the proposed strategy as compared to other strategies. The fitness function is presented as

$$
\begin{aligned}
\eta_{o}= & w_{\mathrm{SMA}}\left(\frac{\mathrm{RMS}_{\mathrm{SMA}}-\mathrm{RMS}_{\mathrm{SMA}-\mathrm{min}}}{\mathrm{RMS}_{\mathrm{SMA}-\min }}\right) \\
& +w_{\mathrm{DTL}}\left(\frac{\mathrm{RMS}_{\mathrm{DTL}}-\mathrm{RMS}_{\mathrm{DTL}-\mathrm{min}}}{\mathrm{RMS}_{\mathrm{DTL}-\min }}\right),
\end{aligned}
$$

where $\eta_{o}$ is the overall performance of the control strategy and $w_{\mathrm{SMA}}$ and $w_{\mathrm{DTL}}$ are the normalization coefficient which can transform the objective RMS value to the certain range $[0,1]$ so as to ensure that each objective RMS value has the same contribution to the fitness function. In this work, $w_{\mathrm{SMA}}$ and $w_{\text {DTL }}$ are equal to each other and set as 0.5 . Then, with the fitness function, the overall performance of each control strategy can be quantified to the same level.

With the above method, the overall performance for each of the control strategies can be calculated. It can be seen from Table 3 that, compared with the ADD strategy, the GHstrategy, and the traditional hybrid-SH and GH-strategy, the overall performance of the proposed strategy is improved by $58.39 \%, 53.66 \%$, and $66.78 \%$, respectively.

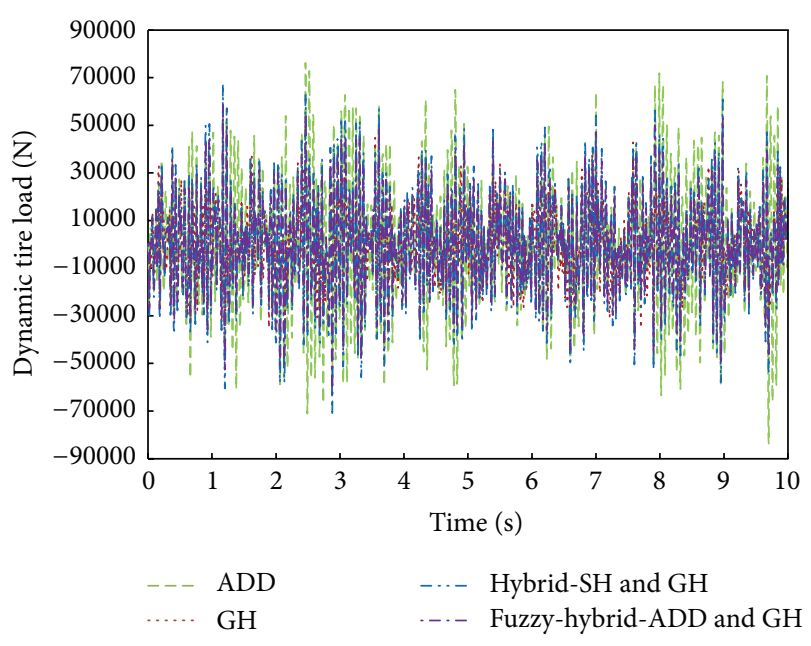

Figure 10: Comparison of dynamic tire load.

\section{Conclusion}

Aiming to improve the ride quality and road friendliness, a fuzzy controlled hybrid-ADD and GH-strategy has been developed for a semiactive suspension system of heavy-duty vehicles. In the design of the proposed control strategy, the fuzzy controller has been successfully employed as the tuning method of the hybrid strategy and was proved to be effective in the coordination of the ADD approach and $\mathrm{GH}$ approach. To examine the performance of the proposed strategy, a quarter-vehicle model, as well as some comparative cases, including sole ADD approach, sole GH approach, and traditional hybrid-SH and GH-strategy, was introduced. Numerical results show that the sole ADD approach and sole GH approach perform best in the SMA and DTL, respectively, while the proposed strategy holds a good performance in the overall performance of the heavy-duty vehicle. In general, this paper provides a new solution for the design of road friendly SAS system of heavy-duty vehicles.

\section{Conflict of Interests}

The authors declare that there is no conflict of interests regarding the publication of this paper. 


\section{Acknowledgment}

The work is supported by the University of Macau Research Grant, Grant nos. MYRG2014-00058-FST, MYRG201400074-FST, and MYRG077(Y1-L2)-FST13-WPK.

\section{References}

[1] M. Valášek, W. Kortüm, Z. Šika, L. Magdolen, and O. Vaculín, "Development of semi-active road-friendly truck suspensions," Control Engineering Practice, vol. 6, no. 6, pp. 735-744, 1998.

[2] D. Karnopp, M. J. Crosby, and R. A. Harwood, "Vibration control using semi-active force generators," Journal of Engineering for Industry, vol. 96, no. 2, pp. 619-626, 1974.

[3] D. Sammier, O. Sename, and L. Dugard, "Skyhook and Hinfinity control of semi-active suspensions: some practical aspects," Vehicle System Dynamics, vol. 39, no. 4, pp. 279-308, 2003.

[4] J. L. Yao, S. Taheri, S. M. Tian, Z. N. Zhang, and L. Shen, "A novel semi-active suspension design based on decoupling skyhook control," Journal of Vibroengineering, vol. 16, no. 3, pp. 1318-1325, 2014.

[5] R. Caponetto, O. Diamante, G. Fargione, A. Risitano, and D. Tringali, "A soft computing approach to fuzzy sky-hook control of semiactive suspension," IEEE Transactions on Control Systems Technology, vol. 11, no. 6, pp. 786-798, 2003.

[6] S. M. Savaresi, E. Silani, and S. Bittanti, "Acceleration-DrivenDamper (ADD): an optimal control algorithm for comfortoriented semiactive suspensions," Journal of Dynamic Systems, Measurement and Control-Transactions of the ASME, vol. 127, no. 2, pp. 218-229, 2005.

[7] L. Balamurugan and J. Jancirani, "An investigation on semiactive suspension damper and control strategies for vehicle ride comfort and road holding," Proceedings of the Institution of Mechanical Engineers. Part I: Journal of Systems and Control Engineering, vol. 226, no. 8, pp. 1119-1129, 2012.

[8] M. Valasek and W. Kortum, "Road-friendly trucks," Structural Dynamics, vol. 1-2, pp. 855-860, 1999.

[9] J.-H. Koo, M. Ahmadian, M. Setareh, and T. M. Murray, "In search of suitable control methods for semi-active tuned vibration absorbers," Journal of Vibration and Control, vol. 10, no. 2, pp. 163-174, 2004.

[10] A. Zin, O. Sename, P. Gaspar, L. Dugard, and J. Bokor, "Robust LPV-H infinity control for active suspensions with performance adaptation in view of global chassis control," Vehicle System Dynamics, vol. 46, no. 10, pp. 889-912, 2008.

[11] L. Flores, A. Drivet, R. A. Ramírez-Mendoza, O. Sename, C. Poussot Vassal, and L. Dugard, "Hybrid optimal control for semi-active suspension systems," in Proceedings of th 10th Mini Conference on Vehicle System Dynamics, Identification and Anomalies (VSDIA '06), pp. 505-512, Budapest University of Technology and Economics, Budapest, Hungary, November 2006.

[12] Z. Xie, P. K. Wong, J. Zhao, T. Xu, K. I. Wong, and H. C. Wong, "A noise-insensitive semi-active air suspension for heavy-duty vehicles with an integrated fuzzy-wheelbase preview control," Mathematical Problems in Engineering, vol. 2013, Article ID 121953, 12 pages, 2013.

[13] S.-B. Choi, D.-W. Park, and M.-S. Suh, "Fuzzy sky-ground hook control of a tracked vehicle featuring semi-active electrorheological suspension units," Journal of Dynamic Systems, Measurement and Control, vol. 124, no. 1, pp. 150-157, 2002.
[14] S. H. Ha, S. B. Choi, G. S. Lee, and W. H. Yoo, "Control performance evaluation of railway vehicle MR suspension using fuzzy sky-ground hook control algorithm," Journal of Physics: Conference Series, vol. 412, Article ID 012042, pp. 1-5, 2013.

[15] Z. C. Xie, P. K. Wong, J. Zhao, and T. Xu, "Design of a denoising hybrid fuzzy-pid controller for active suspension systems of heavy vehicles based on model adaptive wheelbase preview strategy," Journal of Vibroengineering, vol. 17, pp. 883-904, 2015. 


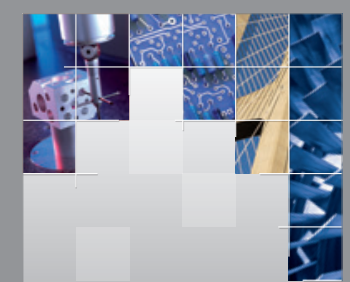

\section{Enfincering}
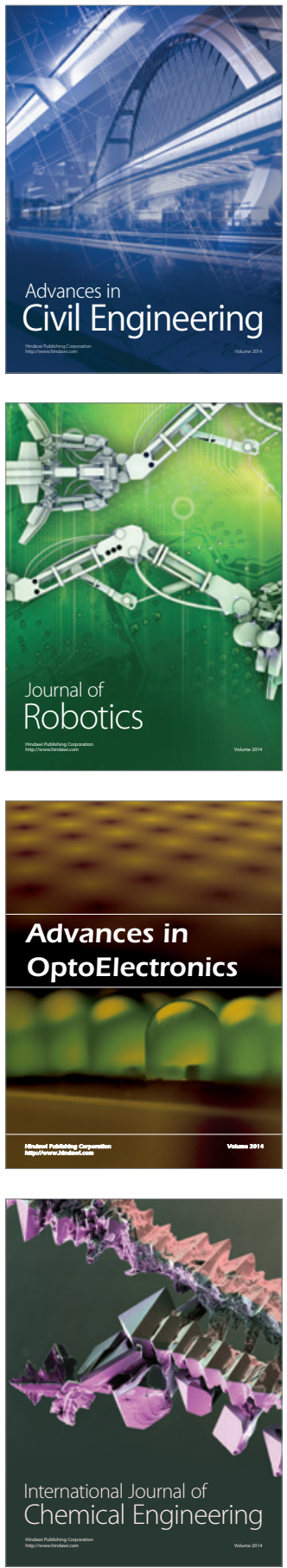

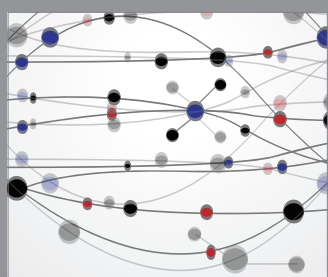

The Scientific World Journal

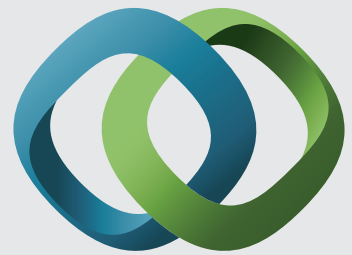

\section{Hindawi}

Submit your manuscripts at

http://www.hindawi.com
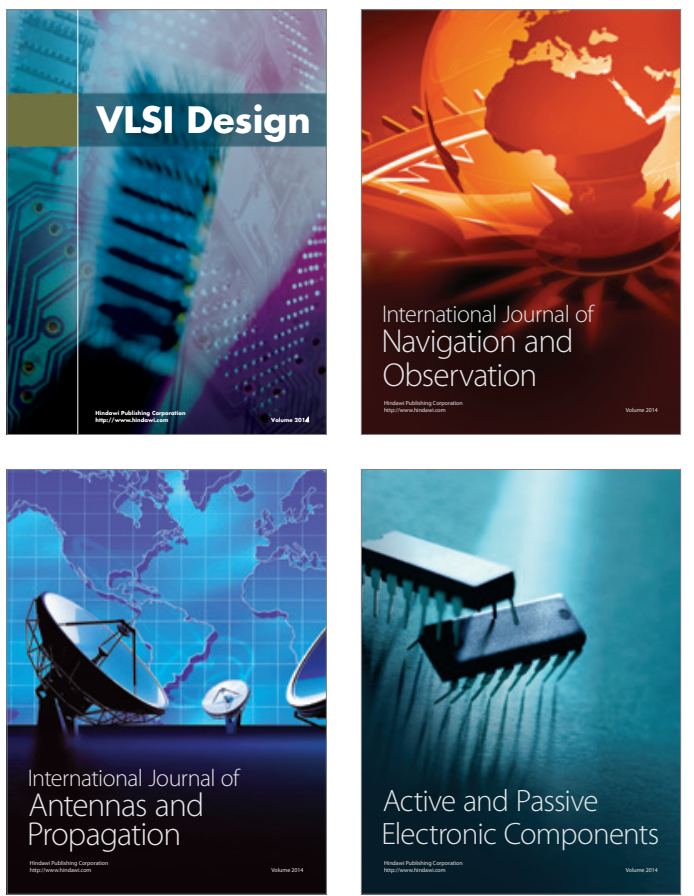
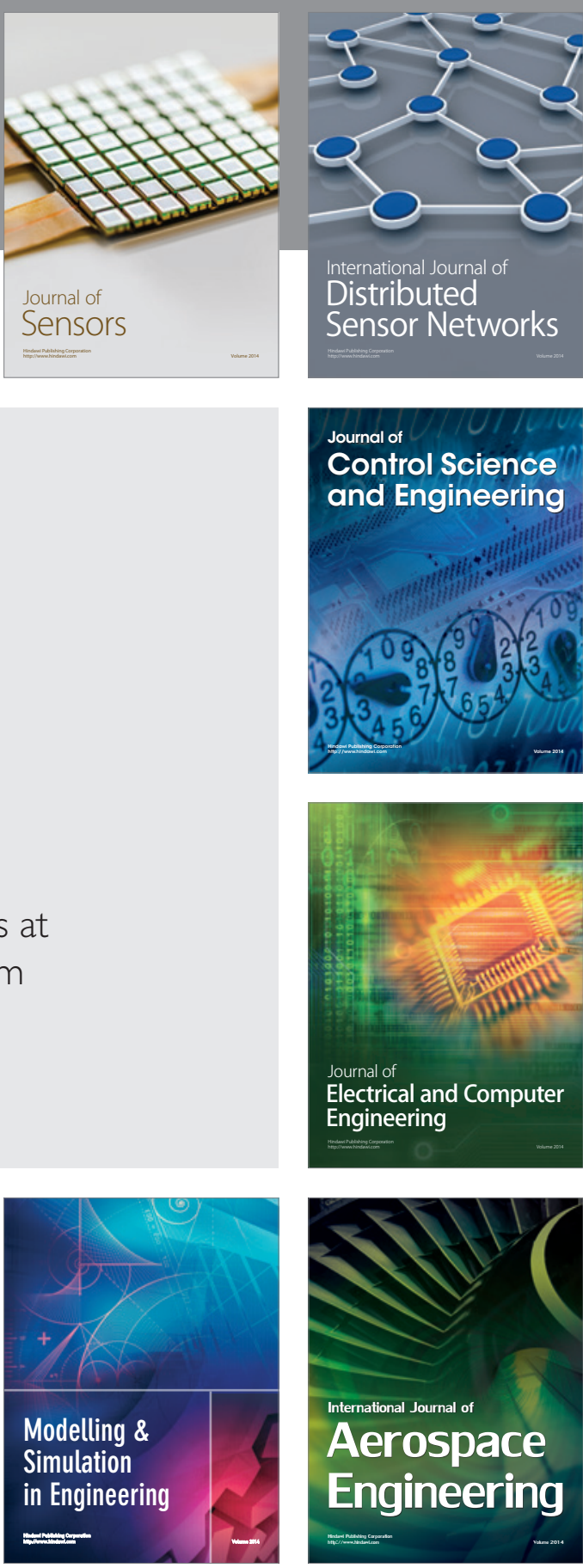

International Journal of

Distributed

Sensor Networks

Journal of

Control Science

and Engineering
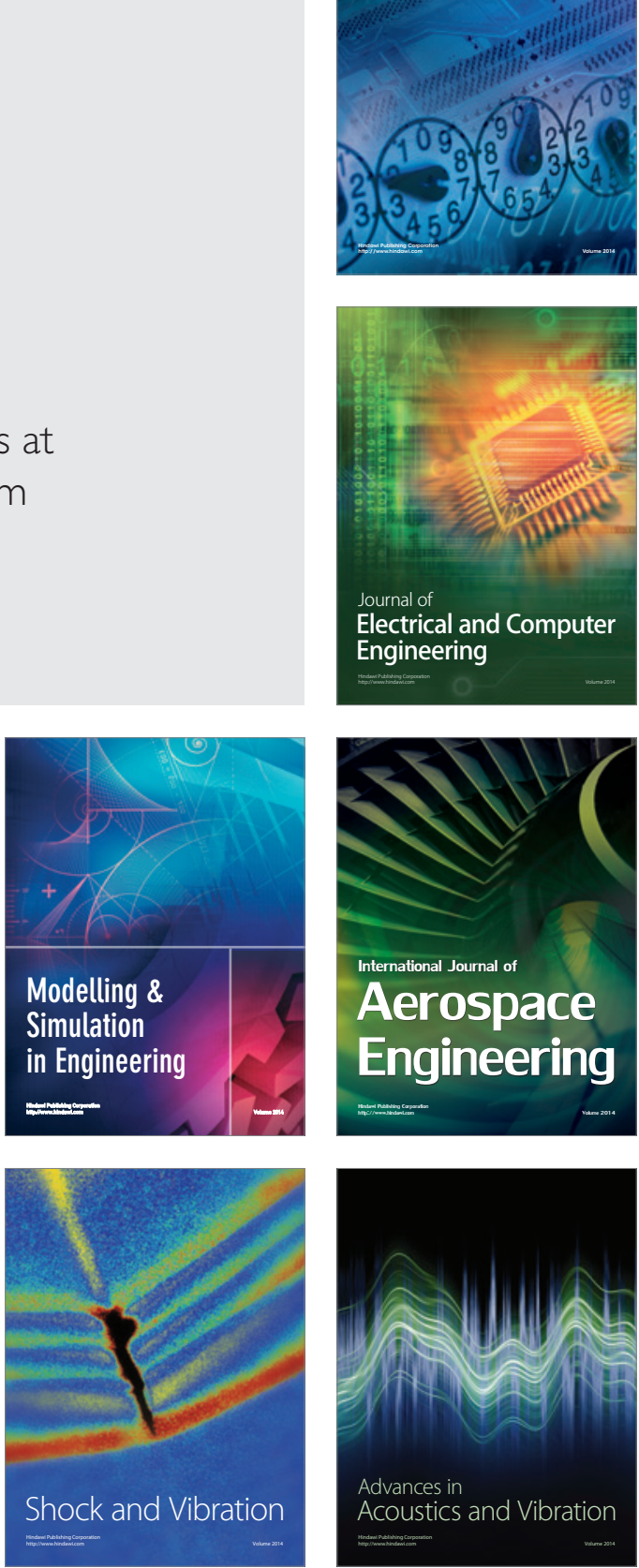